\title{
Computer-aided modeling of aquaculture plants
}

\begin{abstract}
ARNE TYSS $\varphi \dagger$
Keywords: Modeling, simulation, data processing, growth model.

Mathematical modeling of dynamic processes is often considered an intricate and time consuming task. Program packages for simulation, time series analysis and identification in combination with modern data logging equipment allow the task to be handled in a simpler and more efficient way.

This paper gives a short description of the different tools, and one example illustrates how a stochastic growth model and a simulation program can be utilized to simulate the weight distribution of fish in an aquaculture plant as a function of time, temperature and feeding level. The model allows for stochastic variation in all parameters and input variables and may be utilized among plant managers for examination of production strategies and among researchers to study the key variables affecting growth.
\end{abstract}

\section{Introduction}

During the last two decades considerable advances have been made in the development of general, mathematical and computational techniques for process analysis, optimization and control. The application of these techniques to many chemical and system engineering operations has met with considerable success, and they are becoming standard tools in control engineering practice. However, less attention is paid to aquaculture processes where one has to deal with biological systems working in a technical environment.

The major differences between traditional process industry and aquaculture plants are that in general, the former is very much better understood and the availability of process measurements may be taken for granted in the majority of cases. In contrast we are only just beginning to attain a quantitative understanding of aquaculture processes, and lack of robust sensors and measurement methods may force us either to settle for less sophisticated control and monitoring systems or to exert a considerable amount of model-building effort before any work on optimization or control may commence.

Mathematical models and dynamic simulation have long been considered indispensable for the understanding of process dynamics and for control system design. This is particularly true when it comes to plants of a realistic complexity with all process equipment and instrumentation systems included. In such a situation simulation is the only workable way to compare alternative production schedules, control strategies or for training of process operators. By means of simulation you can find answers to questions that could be very expensive to answer through real

Received 15 January 1987.

This paper was presented at the IFAC Symposium on Automation and Dataprocessing in Aquaculture, Trondheim, Norway, 18-20 August 1986.

This paper is reprinted with the permission of IFAC.

$\uparrow$ CAMO A/S, Trondheim, Norway. 
plant experiments, for instance to find optimal rearing conditions and production strategies.

The heart of every simulation system is the model of the process, and very often the engineer does not have the tools available which make the modelling procedure practicable. One way to improve the situation is to utilize interactive programs for simulation, identification and time series analysis.

\section{Use of mathematical models in aquaculture}

To design mathematical models of aquaculture plants requires knowledge of biology, chemistry and physics. In most cases there are close interactions between the biological part and the technical environments, and if these are not taken into account, one may end up with models which are not suitable for control or monitoring purposes.

The biological part is by far the most complicated since one has to deal with living individuals. Models of process equipment like heat exchangers, heat pumps, filters etc. may also be complicated, but the modeling problem has been thoroughly treated in the literature and suitable models are readily available.

Models of growth in rearing tanks and ponds have been designed for a variety of species but in most cases one does not take into account the interactions between the physiological state of the individuals and the physical and chemical state of the environment surrounding the stock. This is not a straightforward task, but for the purpose of deriving models for a population of fish in captivity, radical simplifications must be made.

The modeling procedure is drastically simplified if an interactıve simulation program is available. It is then easy to check different model structures, and see how the choice of assumptions and parameter values influences the model behaviour. The availability of modern computing facilities also removes the traditional thinking that mathematical models have to be simple in order to be useful.

All mathematical modeling activities have to be verified by comparing model responses with responses from the aquaculture plant. This implies that process recordings must be available and in a form that allows straightforward comparison. Unfortunately this is not the situation in today's aquaculture plants because there is a lack of measurement devices and sensors suitable for recording biomasses and environmental variables such as $\mathrm{pH}$ and $\mathrm{O}_{2}$.

A typical Norwegian aquaculture plant is based on rearing salmon in cages in the sea to market size or smolt production in tanks. The main problem is to find optimal growth conditions as a function of the parameters and variables that are controllable. In a tank system it is possible to manipulate most of the input variables (temperature, oxygen, $\mathrm{pH}$, waterflow), but in a cage system these variables are generated from the environment and it is not possible for man to manipulate them.

In a salmon farm the optimal growth conditions are found during the summer and up to a certain upper temperature limit, the growth rate is an increasing function of temperature. The prices for smolt and salmon and food (in the case where waste-fish is used) vary in the course of the year and a maximization of tons of salmon and number of smolt per year, do not necessarily result in the highest profit. Typical questions that need answers are:

What is the optimal rearing temperature?

At what size and age should smolt be delivered? 
When and at which size should the salmon be sold?

How should the limiting growth factors be controlled?

The answers may be found using mathematical models and simulation.

\section{Use of interactive CAD-program packages}

The use of interactive programs for simulation, identification and time series analysis has to a great extent reduced the number of problems involved in mathematical model building. By combining the intuition and experience of the user and the data handling and computing capacity of the computer, the modeling procedure can be more efficiently taken care of. The user does not need to be an expert in programming or computer operating systems.

CYPROS $\uparrow$ (Cybernetic program packages) is an interactive program system which is designed to handle a wide range of engineering problems, not only modeling but also analysis and design of control systems. The program system has been developed over a period of almost ten years, with emphasis on adaptation to industrial processes (Tyssø 1958). The programming language is standard Fortran 77 and the system is run on PC as well as larger computer systems (VAX, ND).

The CYPROS programs are operated through a few commands and a default driven question-answer dialogue. This makes learning and remembering very easy. Available commands are presented on the screen when needed. All the commands are descriptive and can be used in abbreviated forms. In some programs the default command changes as you proceed. This takes you through the working procedure and makes operation fast and easy.

The CYPROS programs are structured to facilitate testing of different strategies. The system configuration, parameters and methods can easily be changed while working with the program in order to study the results under different conditions. Easy communication and fast screen graphics give flexible operation of different command modes.

CYPROS consists of a number of special purpose packages for simulation, identification and control. In addition there are packages for matrix handling and time series analysis and manipulation. Figure 1 illustrates the capabilities of the CYPROS system. In the simulation package three different programs are available for solving differential and difference equations. The most general and powerful tool is SIM. With SIM the user specifies his/her models by means of Fortran subroutines, and changing constants and initial values stored on the data file may easily be done during a simulation run. The simulation results are presented on a colour graphic display and hard copies are easily produced. The most commonly used integration methods are implemented, methods such as Euler and Kutta Merson. SIM may be used in off-line simulation as well as real-time simulation.

The identification package contains programs for identification and parameter estimation. Many powerful techniques are implemented and single input-single output and multivariable, linear and nonlinear models are considered. Methods such as the Maximum Likelihood method and the Extended Kalman filter method are available. By means of the time series analysis program the user can easily

† Developed at the Norwegian Institute of Technology, Div. of Engineering Cybernetics and SINTEF, The Foundation of Scientific and Industrial Research, Dep. of Automatic Control at the University of Trondheim, Norway. 


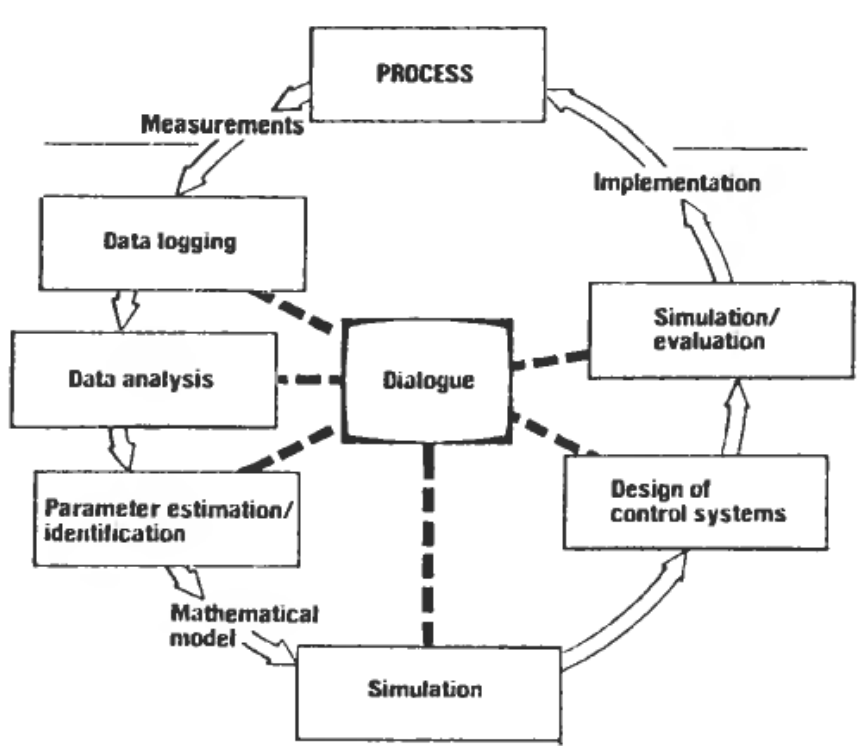

Figure 1. The capabilities of the CYPROS system.

operate on data files (edit, scale, filter) and perform standard statistical analysis (compute correlation functions and power spectra). There is also available a powerful programmable matrix calculator which can handle a great number of different matrix manipulations and operations.

The control package contains a wide variety of design and analysis methods. Adaptive controllers based on the generalized minimum variance algorithm and a pole placement technique are implemented.

\section{Data acquisition system}

Plant recordings are essential in modeling, either to be used for model evaluation or as input data for identification purposes. Data logging is known to involve many practical problems such as scaling, analog/digital converting, and noise filtering, but new developments in computer technology and hardware have led to programmable data logging equipment with capabilities that radically simplify the logging procedure.

Transfer of data from the logging system to the computer is often considered a problem, but can be taken care of in the CYPROS system using three different principles.

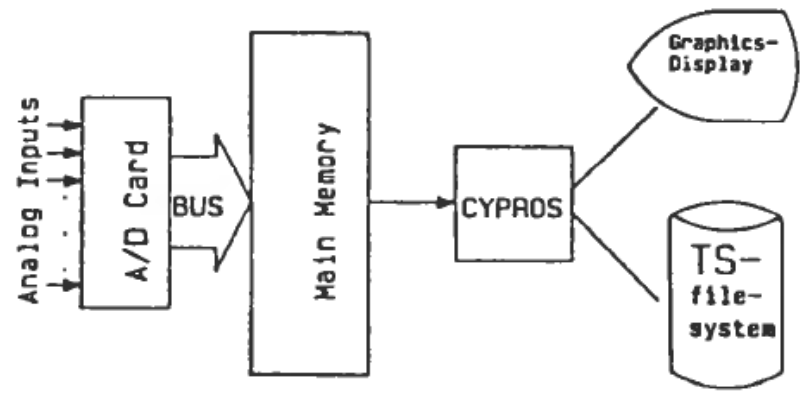

Figure 2. 


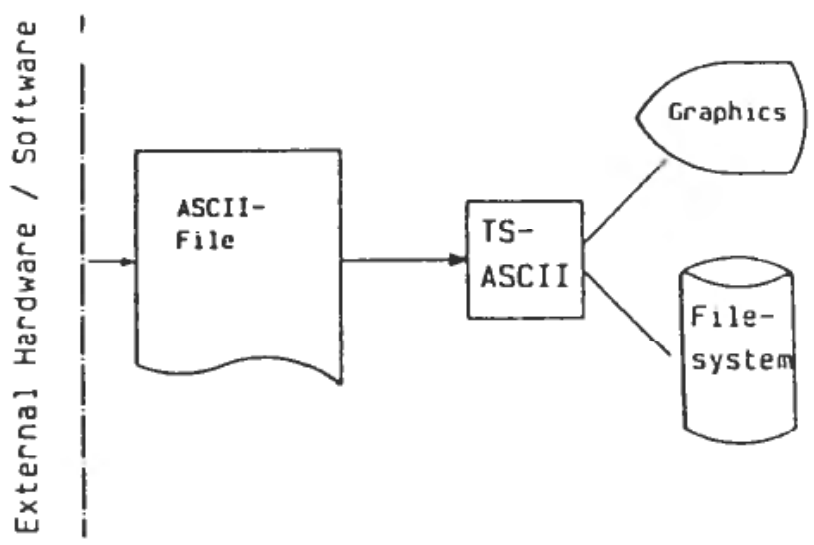

Figure 3.

(a) Using an analog/digital converter card that fits into one of the PC's spare slots. See Fig. 2.

Data may then be transferred in the simplest possible way directly into your PC's main memory, displayed and stored on disk in the CYPROS file format.

This is a simple and effective approach especially where large amounts of data are handled. The setup is excellent for laboratory use, but has obvious limitations in the field.

(b) Import data from a standard ASCII file, see Fig. 3.

Any time series represented in ASCII-format in the CYPROS host computer file system can be translated into the CYPROS file format using a dedicated program. A predefined layout is required.

This is the most flexible solution, as any kind of equipment may be used to record the data and any file transfer system may be used to move data from one computer to another (for example via telephone from another continent, a ship or a marine construction).

(c) Using a portable data logger that the CYPROS input/output system is adapted to. See Fig. 4.

A portable data logger is the best solution for data acquisition in field conditions. Many of these record data on their own media, magnetic tape, CMOS memory etc, but they are also capable of transferring this information to another device via a more or less standard interface.

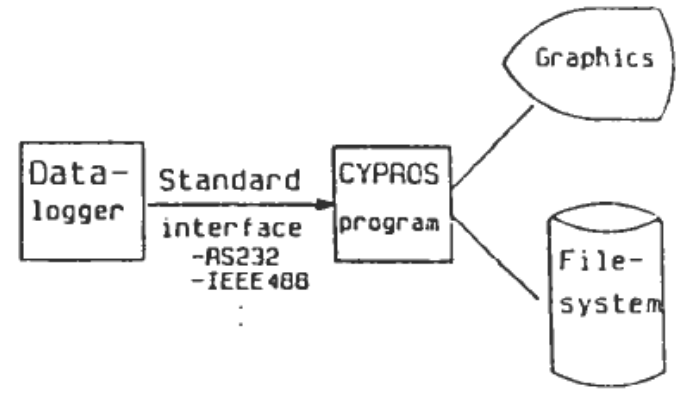

Figure 4. 


\section{A fish growth model}

Starting with a simple material balance equation one may say that the weight increment of a fish is equal to the difference of what is going into and what is coming out of the fish. If, in addition, one assumes that the fish and the food have an approximately equal chemical composition, one may formulate the growth of a single fish as a first order nonlinear differential equation:

$$
\frac{d \omega}{d t}=(1-A) \beta . f . h(T) \omega^{m}-k(T) . \omega^{n}
$$

where

$\omega$ weight of the fish

$T$ temperature

$A$ food producing energy coefficient

$\beta$ assimilation efficiency coefficient

$k(T)$ coefficient of catabolism

$n$ exponent of catabolism

$m$ exponent of anabolism

$h(T)$ coefficient of anabolism

$f$ feeding level, $0 \leqslant f \leqslant 1$

Eqn. (1) is very often referred to in the literature and a detailed description of the model may be found in Sparre (1983) and From and Rasmussen (1984).

The feeding level of each fish may vary from 0 (starving fish) to 1 (fish eating max ration) and must be converted to $\mathrm{kg}$ food per day per fish. Since the waste of food is a poorly known quantity, particularly for open systems in the sea, it would be difficult to specify exact values and one should operate with stochastic variables characterized by mean value, $\mu$ and variance, $\sigma^{2}$. The determination of $f$ from experiments is discussed in Sparre (1983).

The temperature is regarded as a control variable in a tank system and as a disturbance in a cage system. $k(T)$ and $h(T)$ may be determined from experiments, see From and Rasmussen (1984) and up to a certain maximum temperature, $T_{\max }$, one has

$$
\begin{aligned}
& h(T)=h_{1} \exp \left(h_{2} T\right) \\
& k(T)=k_{1} \exp \left(k_{2} T\right)
\end{aligned}
$$

\section{Simulation of growth}

It is well known that aquaculture species like salmon and trout of the same size do not grow at an equal rate, so it is necessary to include some randomness in the growth equation (1). This can be taken care of by describing the input variables or some of the growth parameters as stochastic processes. Equation (1) is easily solved using the simulation program SIM, and Fig. 5 shows typical growth curves for different values of the assimilation coefficient $\beta$. $\beta$ is assumed to be a stochastic variable (Gaussian) with mean value $\bar{\beta}=0.8$ and variance $\sigma_{\beta}^{2}=0 \cdot 17^{2}$. The initial weight of each fish is $275 \mathrm{~g}$, feeding level, $f=0.9, A=0.25, T=10^{\circ} \mathrm{C}, m=0.7$, $n=0.7, h_{1}=0.06, h_{2}=0.12, k_{1}=0.007, k_{2}=0.044$. The simulation period is 80 days. 


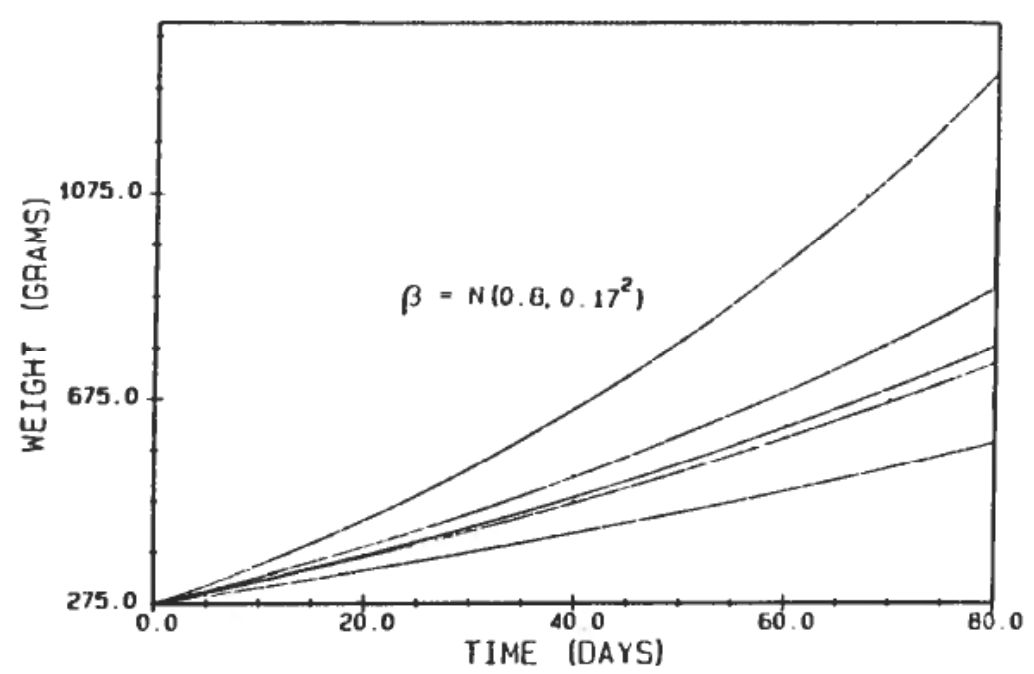

Figure 5.

Given a certain weight distribution at the beginning of a growth period, the problem is to find the weight distribution at the end of the growth period. During a growth period it is assumed that the feeding level, temperature and other input variables are kept constant or have a constant mean value. The number of growth periods during a year is dependent upon the rearing conditions and may vary from a few days up to several weeks or months. The weight of an individual can only be given with a certain degree of accuracy so it is natural to divide all individuals into weight groups.

In the examples shown below it is assumed that the stock consists of ten weight groups, $M=10$ where the groups are as follows:

$$
\begin{array}{ccc}
M_{1}=250 \mathrm{~g}-300 \mathrm{~g}, & M_{2}=300 \mathrm{~g}-350 \mathrm{~g}, & M_{3}=350 \mathrm{~g}-425 \mathrm{~g}, \\
M_{4}=425 \mathrm{~g}-500 \mathrm{~g}, & M_{5}=500 \mathrm{~g}-600 \mathrm{~g}, & M_{6}=600 \mathrm{~g}-700 \mathrm{~g}, \\
M_{7}=700 \mathrm{~g}-825 \mathrm{~g}, & M_{8}=825 \mathrm{~g}-950 \mathrm{~g}, & M_{9}=950 \mathrm{~g}-1100 \mathrm{~g}, \\
\text { and } M_{10}=1100 \mathrm{~g}-1250 \mathrm{~g} &
\end{array}
$$

If the number of individuals in each group, $j$, is $y_{j}$, then one may describe the weight distribution during a growth period by the vector

$$
\mathbf{y}^{T}=\left[y_{1}, y_{2} \ldots y_{10}\right]
$$

Starting with an initial weight distribution $\mathbf{y}_{0}\left(t_{0}\right)$ at time $t_{0}$ one may apply Eqn. 1 to each individual to see how the weight distribution varies with time.

Figures 6-7 show the characteristic behaviour. The initial distribution was

$$
\mathbf{y}^{T}\left(t_{0}\right)=[500,0,0,0,0,0,0,0,0,0]
$$

and specifying $\beta$ to be $N\left(0 \cdot 8,0 \cdot 17^{2}\right)$ the result will be as shown in Fig. 6 . The distribution is shown after 11 days and 50 days. Figure 7 shows the result using the same operating conditions, but in this case also the feeding level, $f$, is assumed to be a stochastic variable, $N\left(0 \cdot 9,0 \cdot 08^{2}\right)$.

Every individual in each weight group follows the Markov assumption which briefly says that every individual has the same probability to achieve a prescribed 


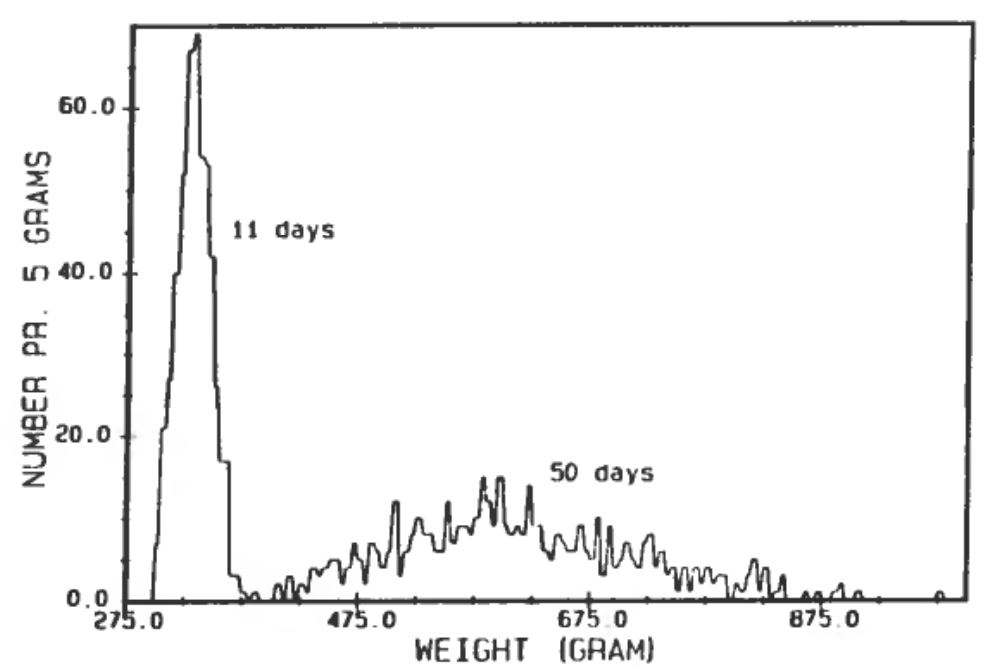

Figure 6.

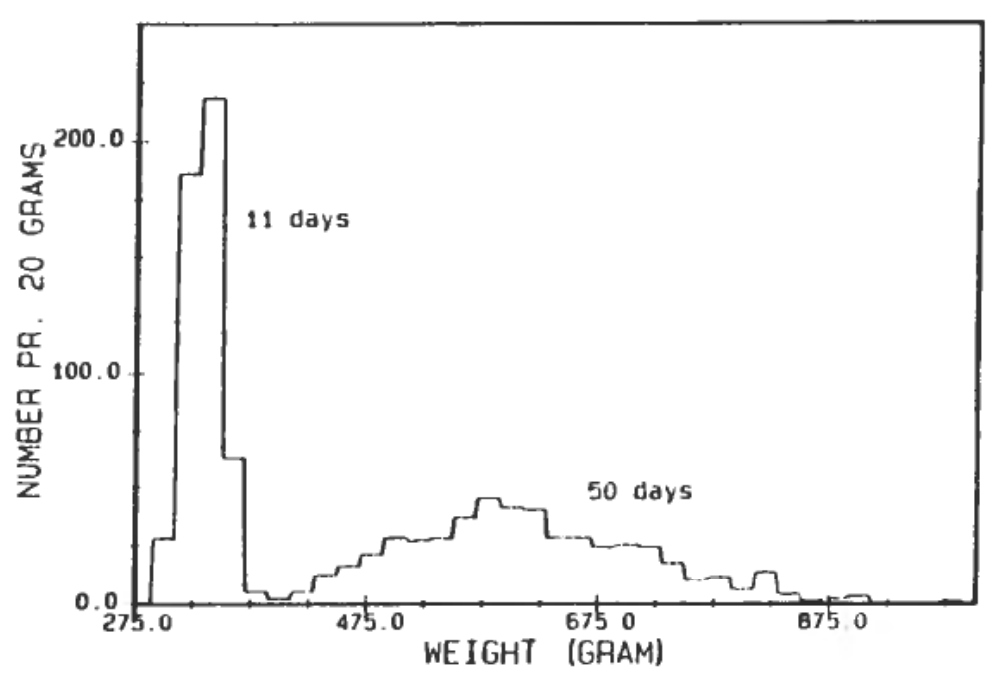

Figure 7.

final weight, independently of how the individual achieved its present weight. This indicates that growth rate should be related to size independent of age.

Due to diseases, predators and escape from the cage system there will always be a certain decrease in the stock. Therefore one has to take into account that only a certain percentage of the stock will survive for a certain period. Mortality may be a function of size and the length of the growth period, and can be taken into account in different ways during a simulation run.

In order to simulate the growth of a population one has to divide the stock into weight groups and use eqn. (1) within each growth period. During a growth period the feeding level and temperature are kept at constant mean values. One individual that during a growth period leaves the weight group it started in, will in the next growth period have an initial weight equal to the mean value of that weight group. If one or more individuals do not leave the original weight group, they will have an increment in initial weight for the next growth period corresponding to the mean 
value of the lack of weight that should have brought them into the next weight group.

Mortality may be related to a low growth rate so that a certain percentage of those individuals that stay in the original weight group will die. Let $\mathbf{x}(t)$ be the state vector where the elements are the number of individuals in each weight group. The transition from one growth period to the other could then be described by

$$
\mathbf{x}_{k+1}=\Phi \mathbf{x}_{k}
$$

where $\Phi$ is the transition matrix whose elements are functions of temperature, feeding level and death rate.

Only in special cases is one able to find numerical expressions for the transition matrix elements see Sparre (1983) but one may solve eqn. (4) using simulation. For each individual one applies eqn. 1 letting $f$ or $\beta$ being a stochastic variable, and at the end of the growth period one updates every weight group according to the rules indicated above. To illustrate how eqn. 4 may be solved by means of simulation we consider the growth of a stock of 2110 individuals over six growth periods where each period is 11 days. The stock distribution at time, $t=0, \mathbf{x}\left(t_{0}\right)$ is:

$$
\mathbf{x}\left(t_{0}\right)^{T}=[500,1000,400,200,10,0,0,0,0,0]
$$

The feeding level is assumed constant in each growth period while the assimilation coefficient, $\beta$ increases with weight as follows (subscripts refer to weight group).

$$
\begin{aligned}
& \beta_{1}=N\left(0 \cdot 7,0 \cdot 27^{2}\right), \quad \beta_{2}=N\left(0 \cdot 76,0 \cdot 21^{2}\right) \\
& \beta_{3}=N\left(0 \cdot 81,0 \cdot 17^{2}\right), \quad \beta_{4}=N\left(0 \cdot 84,0 \cdot 15^{2}\right) \text { and } \\
& \beta_{5}=\beta_{6}=\beta_{7}=\beta_{8}=\beta_{9}=\beta_{10}=N\left(0 \cdot 85,0 \cdot 14^{2}\right) .
\end{aligned}
$$

Mortality decreases with weight and is accounted for by saying that a certain percentage of the individuals that stay in the original weight group during a growth period (low $\beta$ ), dies. In the example the percentage is $5 \%$. The result of simulation is plotted in Fig. 8 where the weight distribution is shown after the first growth period (11 days) and the last period (66 days).

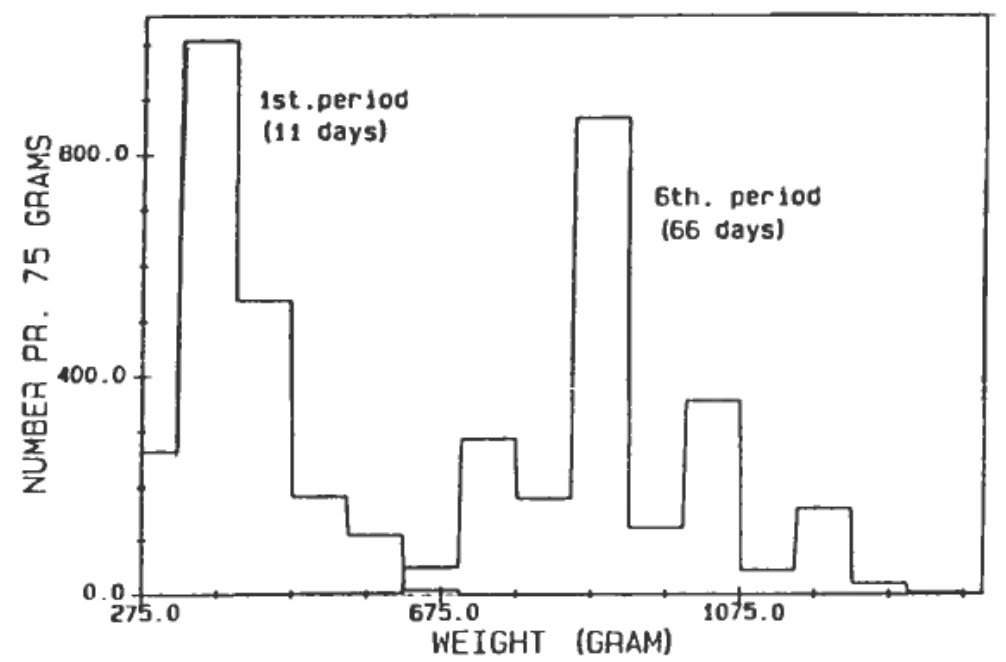

Figure 8. 


\begin{tabular}{|c|c|c|c|c|c|c|c|c|c|c|}
\hline \multirow[b]{2}{*}{ To } & \multicolumn{10}{|c|}{ From } \\
\hline & 1 & 2 & 3 & 4 & 5 & 6 & 7 & 8 & 9 & 10 \\
\hline 1 & 70 & 0 & 0 & 0 & 0 & 0 & 0 & 0 & 0 & 0 \\
\hline 2 & 375 & 82 & 0 & 0 & 0 & 0 & 0 & 0 & 0 & 0 \\
\hline 3 & 55 & 894 & 63 & 0 & 0 & 0 & 0 & 0 & 0 & 0 \\
\hline 4 & 0 & 24 & 328 & 12 & 0 & 0 & 0 & 0 & 0 & 0 \\
\hline 5 & 0 & 0 & 9 & 188 & 0 & 0 & 0 & 0 & 0 & 0 \\
\hline 6 & 0 & 0 & 0 & 0 & 10 & 0 & 0 & 0 & 0 & 0 \\
\hline 7 & 0 & 0 & 0 & 0 & 0 & 0 & 0 & 0 & 0 & 0 \\
\hline 8 & 0 & 0 & 0 & 0 & 0 & 0 & 0 & 0 & 0 & 0 \\
\hline 9 & 0 & 0 & 0 & 0 & 0 & 0 & 0 & 0 & 0 & 0 \\
\hline 10 & 0 & 0 & 0 & 0 & 0 & 0 & 0 & 0 & 0 & 0 \\
\hline 11 & 0 & 0 & 0 & 0 & 0 & 0 & 0 & 0 & 0 & 0 \\
\hline
\end{tabular}

Table 1. Transitions.

Table 1 illustrates how the transition mechanism works out after the first growth period, or

$$
\mathbf{x}_{1}=\Phi \mathbf{x}_{0}
$$

As can be read from the table, 70 of the individuals in group 1 stay in group 1 while for instance group 3 has got 55 new members from group 1, 894 from group 2, while 63 have not succeeded in leaving the original weight group 3.

As can be seen from the Table 2,12 individuals have died during the first growth period, 4 from group 1 and 2, 3 in group 3 and 1 in group 4.

\section{Conclusions}

Computer based tools for mathematical modeling are discussed, and it is shown how, by means of simulation, a better understanding of the growth process in an aquaculture plant is achieved. Improved knowledge about the growth process may lead to more efficient operation and control strategies and thus increased productivity.

\section{REFERENCES}

From, J., and Rasmussen, G. (1984). A model of fish growth in aquaculture. Danish Trout Research Station, Brøns, DK-6780, Skarbæk.

SpARre, P. (1983). Fish Farm Optimization. Danish Trout Culture Research Station, Brøns, DK-6780.

Tyssø, A. (1986). CYPROS, System Description. CAMO A/S, P.O. Box 5115, 7001 Trondheim, Norway.

\begin{tabular}{ccccc}
\hline From & Group & Small & Dead & Rest \\
\hline 1 & 70 & 4 & 66 \\
2 & 82 & 4 & 78 \\
3 & 63 & 3 & 60 \\
4 & 12 & 1 & 11 \\
\hline
\end{tabular}

Table 2. Unsuccessful transitions/deads. 